\title{
REMOVABLE SETS FOR HOLOMORPHIC FUNCTIONS OF SEVERAL COMPLEX VARIABLES
}

\author{
EDGAR LEE STOUT*
}

\begin{abstract}
We show that every closed subset of $C^{N}$ that has finite $(2 N-2)$-dimensional measure is a removable set for holomorphic functions, and we obtain a related result on the ball.
\end{abstract}

\section{Introduction}

A colleague has remarked that Everybody knows that a set too small to be a variety is removable. The present paper is devoted to an explication of certain cases of this general philosophy, which are motivated by a result of Shiffman [11], [12], to the effect that a closed subset $E$ of a domain $\Omega$ in $C^{N}$ is removable for holomorphic functions in the sense that if $f \in \mathcal{O}(\Omega \backslash E)$, then $f$ extends holomorphically to an $\tilde{f} \in \mathcal{O}(\Omega)$ provided $\Lambda^{2 N-2}(E)=0, \Lambda^{2 N-2}$ denoting $(2 N-2)$-dimensional Hausdorff measure. $\dagger$ Because of the Hartogs phenomenon, this result is of interest only in the case that the set $E$ is not compact. Our principal result is an extension of this theorem, in the case that $\Omega$ is $\mathbb{C}^{N}$ itself, that replaces the hypothesis that $\Lambda^{2 N-2}(E)=0$ by the hypothesis that $\Lambda^{2 N-2}(E)$ be finite.

\section{The main result}

We shall prove the following result.

1. Theorem. If $E \subset \mathbb{C}^{N}, N \geq 2$, is a closed set with $\Lambda^{2 N-2}(E)<\infty$, then $E$ is removable.

This is a global theorem in that the conclusion fails for closed sets in bounded domains. For example, if $\Omega$ is a bounded domain that contains the origin, and if $E=\Omega \cap\left\{z_{N}=0\right\}$, then $\Lambda^{2 N-2}(E)<\infty$, but $E$ is not removable, as the function $f(z)=z_{n}^{-1}$ shows.

* Research supported in part by grant DMS-8801032 from the National Science Foundation. fA version of the result of Shiffman had been found earlier by Caccioppoli [3]. 
Proof of the Theorem: We give a direct proof in the case of $\mathbb{C}^{2}$ and then argue by induction.

The proof in $\mathrm{C}^{2}$ depends on a lemma, which is based on work of Alexander [1].

Denote by $\mathbf{B}_{N}$ the unit ball in $\mathbf{C}^{N}$ and by $r \mathbf{B}_{N}$ the set $\left\{r z: z \in \mathbf{B}_{N}\right\}$ when $r \in(0, \infty)$. The boundary $b r \mathrm{~B}_{N}$ is the sphere in $\mathbb{C}^{N}$ of radius $r$ centered at the origin.

The referee has kindly drawn the author's attention to Théorme 4, p. 309, of Sibony's paper [15], which contains this lemma, with the constant 2 rather than the constant $\sqrt{2} \pi$, as a special case. It would be of interest to what the best value of the constant is.

2. Lemma. If $Y$ is a closed subset of br $\mathrm{B}_{2}$ and if the polynomially convex hull of $Y$ contains the origin, then $\Lambda^{1}(Y) \geq \sqrt{2} \pi r$.

Proof: First, let $X \subset b \mathbf{B}_{2}$ be a compact set with $0 \in \hat{X}, \hat{X}$ the polynomially convex hull of $X$. According to Theorem 1 of [1], if $\pi_{j}: C^{2} \rightarrow C$ is the projection given by $\pi_{i}\left(z_{1}, z_{2}\right)=z_{i}, i=1,2$ then

$$
\Lambda^{2}\left(\pi_{1}(\hat{X})\right)+\Lambda^{2}\left(\pi_{2}(\hat{X})\right) \geq \pi
$$

whence one of the summands, say the first, in (1) is at least $\pi / 2$.

Let $Z$ denote the polynomially convex hull of the set $\pi_{1}(\hat{X})$, i.e., the union of $\pi_{1}(\hat{X})$ and the bounded components of $C \backslash \pi_{1}(\hat{X})$. The boundary of $Z$ is the boundary of the unbounded component of the set $C \backslash \pi_{1}(\hat{X})$, and the set $Z$ does not disconnect the plane. According to the isoperimetric inequality [2, $\S \S 14.3,14.6]$

$$
\Lambda^{1}(b Z) \geq 2 \sqrt{\pi}\left[\Lambda^{2}(Z)\right]^{\frac{1}{2}} .
$$

Every point of $b Z$ is a peak point for the algebra $\mathcal{P}(Z),{ }^{*}$ and so for every point $p \in b Z$, the set $\pi_{1}^{-1}(p) \cap \hat{X}$ is a peak set for the algebra $\mathcal{P}(\hat{X})$, which can be identified with $\mathcal{P}(X)$. Consequently, the set $\pi_{1}^{-1}(p)$ meets the Silov boundary for $\mathcal{P}(X)$, i.e., the set $X$ : We have that $\pi_{1}(X) \supset b Z$. As $\pi_{1}$ is a Lipschitz map with Lipschitz constant one, we must have $\Lambda^{1}(X) \geq \Lambda^{1}(b Z)$. As $\Lambda^{1}(b Z) \geq \sqrt{2} \pi$, we have $\Lambda^{1}(X) \geq \sqrt{2} \pi$.

If now $Y \subset b r \mathbf{B}_{2}$, define $T: \mathbb{C}^{2} \rightarrow \mathbb{C}^{2}$ by $T z=r^{-1} z$, and set $X=T Y$. If $0 \in \hat{Y}$, then $0 \in \hat{X}$, so $\Lambda^{1}(X) \geq \sqrt{2} \pi$ whence $\Lambda^{1}(Y) \geq \sqrt{2} \pi r$, and the lemma is proved.

The theorem, in case $N=2$, is proved as follows. Fix a point $z_{0} \in E$; we prove that if $f \in \mathcal{O}\left(C^{2} \backslash E\right)$, then $f$ extends holomorphically into a neighborhood

* We use the customary notation that $\mathcal{P}(S)$ denotes the algebra of continuous functions on the compact set $S$ that can be approximated uniformly by holomorphic polynomials. 
of $z_{0}$. Without loss of generality, we can take $z_{0}$ to be the origin. Let $\rho: \mathrm{C}^{2} \rightarrow$ $[0, \infty)$ be the map $\rho(z)=|| z||=\sqrt{\left|z_{1}\right|^{2}+\left|z_{2}\right|^{2}}$. According to $[2, \S 13.3 ; 4]$, we have

$$
\int_{(0, \infty)}^{*} \Lambda^{1}\left(E \cap \rho^{-1}(z)\right) d t \leq \text { const. } \Lambda^{2}(E)<\infty .
$$

This implies the existence of $t_{j} \in(0, \infty)$ with $t_{1}<t_{2}<\ldots, t_{j} \rightarrow \infty$, such that

$$
\lim _{j \rightarrow \infty} \Lambda^{1}\left(E \cap b t_{j} \mathbf{B}_{2}\right)=0
$$

Fix a value of $j$ large enough that $t_{j}>1$ and $\Lambda^{1}\left(E \cap b t_{j} \mathbf{B}_{2}\right)<1$.

The lemma implies that the origin does not lie in the polynomially convex hull of the set $E \cap b t_{j} \mathrm{~B}_{2}$. If $\Phi_{j}$ denotes the restriction to $b t_{j} \mathrm{~B}_{2} \backslash E$ of the function $f$, then $\Phi_{j}$ satisfies the tangential Cauchy-Riemann equations and so $([6],[7],[8$, Appendix $])$ continues holomorphically into $t_{j} \mathbf{B}_{2} \backslash\left(E \cap b t_{j} \mathbf{B}_{2}\right)^{\wedge}$, which is a neighborhood of the origin. Denote this extension by $\tilde{\Phi}_{j}$. That $\tilde{\Phi}_{j}$ is an extension of $f$ follows from the fact that $f$ and $\tilde{\Phi}_{j}$ agree on an open subset of $b t_{j} \mathbf{B}_{2}$.

The theorem is proved now in the two-dimensional case. We next assume it proved in the $N$-dimensional case and derive the $(N+1)$-dimensional case. To this end, it is of some importance to notice that the argument just given works equally well granted only that $\Lambda^{2}(E \cap\{z:|z|>1\})$ is finite.

We consider in $C^{N+1}$ a closed subset $E$ with $\Lambda^{2 N}(E)<\infty$. Let $f \in$ $\mathcal{O}\left(\mathcal{C}^{N} \backslash E\right)$. Fix a point $z \in \mathbb{C}^{N+1}$ and denote by $\mathcal{G}_{N+1, N}(z)$ the Grassmannian of all complex affine $\mathrm{N}$-planes in $\mathrm{C}^{N+1}$ that pass through the point $z$. There is a natural invariant measure on $\mathcal{G}_{N+1, N}(z)$, which we shall denote by $d \mu(\Pi)$. We assume this measure to be normalized so that it has total mass one. We have by [12] that if $\widetilde{E}=E \cap\{|z| \geq 1\}$, then

$$
\int_{\mathcal{G}_{N+1, N}}^{*} \Lambda^{2 N-2}(\tilde{E} \cap \Pi) d \mu(\Pi)<c_{N} \Lambda^{2 N}(\tilde{E})<\infty
$$

for a fixed constant $c_{N}$. In particular, for almost every II $\in \mathcal{G}_{N+1, N}(z), \Lambda^{2 N-2}$ $(\tilde{E} \cap \Pi)<\infty$. Thus, for almost every $\Pi, f \mid(\Pi \backslash E)$ extends holomorphically through all of $\Pi$. Denote this extension by $f_{\Pi, z}$. We define

$$
F(z)=f_{\Pi, \Sigma}(z) .
$$

This gives a well-defined value for $F(z)$, because $f_{\Pi, z}(z)$ is independent of the choice of $\Pi$ : Two $\Pi$ 's, say $\Pi_{1}$ and $\Pi_{2}$, in $\mathcal{G}_{N+1, N}(z)$ intersect in an affine subspace of $C$ of positive dimension on which $f_{\Pi_{2}, z}$ and $f_{\Pi_{2}, z}$ agree. Thus they agree at $z$. The function $F$ defined in this way is defined on all of $\varepsilon^{N+1}$, and it agrees with $f$ on $C^{N+1} \backslash E$. 
We have to see that $F$ is holomorphic, and for this, it suffices to show that it is continuous. To do this, let $\left\{z_{n}\right\}_{n=1}^{\infty}$ be a sequence in $C^{N}$ that converges to $z_{0}$; we shall show that $F\left(z_{n}\right) \rightarrow F\left(z_{0}\right)$. Fix a $\Pi_{0} \in \mathcal{G}_{N+1, N}\left(z_{0}\right)$ with $\Lambda^{2 N-2}\left(\Pi_{0} \cap E\right)<\infty$ and such that, $F$ is holomorphic on $\Pi_{0}$. For each $n$, choose $\Pi_{n} \in \mathcal{G}_{N+1, N}\left(Z_{n}\right)$ such that $\Lambda^{2 N-2}\left(\Pi_{n} \cap E\right)<\infty$, such that $F$ is holomorphic on $\Pi_{n}$, and such that $\Pi_{n} \rightarrow \Pi_{0}$.

If $z \in C^{N}$ and $\Pi \in \mathcal{G}_{N+1, N}(z)$, denote by $\mathrm{P}_{z}(\Pi)$ the projective space of all complex lines in $\Pi$ through the point $z$. We have $\operatorname{dim}_{\mathbf{R}} \mathbf{P}_{z}(I)=2 N-2$. There are large values of $R$ such that $\Lambda^{2 N-3}\left(b B_{N+1}(z, R) \cap E \cap \Pi_{0}\right)<\infty$, so if $\pi$ : $\Pi_{0} \backslash\left\{z_{0}\right\} \rightarrow \mathrm{P}_{z_{0}}\left(\Pi_{0}\right)$ is the standard projection, then $\pi\left(b \mathrm{~B}_{N+1}\left(z_{0}, R\right) \cap E \cap \Pi_{0}\right)$ is a set of measure zero in $P_{z_{0}}$. Thus, there is a complex line $\lambda_{0}$ with $z_{0} \in \lambda_{0} \subset$ $\Pi_{0}$ and with $\lambda_{0} \cap E \cap b \mathrm{~B}_{N+1}\left(z_{0}, R\right)=\emptyset$. We may choose $\lambda_{n} \in \mathrm{P}_{z_{n}}\left(\Pi_{n}\right)$ so that $\lambda_{n} \rightarrow \lambda_{0}$. For large values of $n, \lambda_{n} \cap E \cap b \mathbf{B}_{N+1}\left(z_{0}, R\right)=\emptyset$. If we apply the Cauchy integral formula in $\lambda_{n}$ and $\lambda_{0}$ to represent $F\left(z_{n}\right)$ and $F\left(z_{0}\right)$ as the Cauchy integral of $f$ over the circle $\lambda_{n} \cap b \mathbf{B}_{n+1}\left(z_{0}, R\right)$ and of $\lambda_{0} \cap b \mathbf{B}_{N+1}\left(z_{0}, R\right)$, respectively, we find that as $n \rightarrow \infty, F\left(z_{n}\right) \rightarrow F\left(z_{0}\right)$ as desired.

Thus, $F$ is continuous and so necessarily holomorphic.

This completes the proof of the theorem.

\section{Variations on the theme}

The first variation is to the effect that there is an analogue of the result for submanifolds of $\mathrm{C}^{N}$ : Let $\mathcal{M}$ be a k-dimensional complex submanifold of $\mathrm{C}^{N}$, and let $E \subset \mathcal{M}$ be a closed subset with $\Lambda^{2 k-2}(E)<\infty$. If $f \in \mathcal{O}(\mathcal{M} \backslash E)$, then $f$ continues holomorphically into all of $M$.

In the case that $\mathcal{M}$ is an algebraic manifold, we can invoke $[10$, Th. 10, p. 52] to find a projection $\pi: C^{N} \rightarrow C^{k}$ that exhibits $M$ as an analytic cover over $C^{k}$. Using symmetric functions and applying the result already established in $\mathrm{C}^{k}$, we can derive the result on $\mathcal{M}$.

In the case of a general $\mathcal{M}$, there will be no such projection, and, in essence, it is necessary simply to rewrite the proof given above. The case $n=2$ proceeds as before: Fix $z_{0} \in E$. For certain large values of $t, \Lambda^{ \pm}\left(E \cap b \mathbf{B}_{N}\left(z_{0}, t\right)\right)$ will be small and $b \mathrm{~B}_{N}\left(z_{0}, t\right) \cap \mathcal{M}$ will be a smooth $(2 k-1)$-dimensional real hypersurface that bounds the domain $\Delta\left(t, z_{\theta}\right)=\mathbf{B}_{N}\left(z_{0}, t\right) \cap \mathcal{M}$. By Lemma 2 , the polynomially convex hull of $E \cap b \mathrm{~B}_{N}\left(z_{0} t\right)$ does not contain $z_{0}$, and by the extension theorem given by Laurent-Thiebeaut [6], $f \mid b \Delta\left(t, z_{0}\right) \backslash E$ extends holomorphically into a neighborhood of $z_{0}$. The rest of the argument in the two-dimensional case is as before.

For the induction step we replace the affine hyperplanes used in the proof of the theorem by intersections $\mathcal{M} \cap \Pi, \Pi$ a codimension one affine hyperplane

"Here, as above, we are computing Hausdorff measures with respect to the Euclidean metric on $C^{N}=\mathbf{R}^{2 N}$. Below we shall consider the Hausdorf measures associated to certain other metrics, but there we shall be quite explicit about the metrics involved. 
in $\mathbb{C}^{N}$ that is transverse to $M$. The generic $I I$ is transverse to $M$ and so, generically, $\mathcal{M} \cap \Pi$ is a codimension one submanifold of $\mathcal{M}$. In a bit more detail, if $z \in \mathcal{M}$, then almost every $\Pi \in \mathcal{G}_{N, N-1}(z)$ is transverse to $\mathcal{M}$ and, by $\left[12\right.$, Lemma 5] almost every $\Pi$ also satisfies $\Lambda^{2 k-4}(\Pi \cap E)<\infty$. Thus, the induction hypothesis applies to extend $f(\mathcal{M} \cap \Pi \backslash E)$ to an $f_{\Pi} \in \mathcal{O}(\mathcal{M} \cap \Pi)$. We define $F(z)=f_{\Pi}(z)$; this is well-defined and gives the desired extension of $f$ throughout $M$.

A second variation of the theme is that the hypothesis that $\Lambda^{2 N-2}(E)$ be finite can be replaced by the condition that $\Lambda^{2 N-2}\left(E \cap r \mathrm{~B}_{N}\right)$ not grow too rapidly as a function of $r, r \rightarrow \infty$. In fact if $E$ is a closed subset of $\mathbb{C}^{2}$ that satisfies $\Lambda^{2}\left(E \cap r \mathrm{~B}_{N}\right)<\alpha r^{2}$ for all large $r$, then $E$ is removable provided $\alpha<\frac{\pi^{2}}{4 \sqrt{2}}$.

That the desired conclusion can be drawn may be seen as follows. Notice first that $\Lambda^{2 N-2}\left(E \cap r \mathbf{B}_{N}\right)<\alpha r^{2}$ for large $r$ implies that $\Lambda^{2 N-2}\left(E \cap \mathbf{B}_{N}(p, r)\right)<$ $\alpha r^{2}$ for large $r$, no matter what center $p$ is chosen. Next, we have by $[2,4]$ that

$$
\alpha r^{2}>\Lambda^{2}\left(E \cap r \mathbf{B}_{N}\right) \geq \frac{\pi}{4} \int_{[0, r]}^{*} \Lambda^{1}(E \cap\{|z|=t\}) d t .
$$

Consequently,

$$
\Lambda^{1}(E \cap\{|z|=t\})<\sqrt{2} \pi t
$$

for infinitely many arbitrarily large values of $t$, and this implies that the origin is not in the polynomially convex hull of $E \cap\{|z|=t\}$ for such values of $t$. Thus, by arguments we have used already, $f$ continues holomorphically into a neighborhood of the origin. Similarly, it continues holomorphically into a neighborhood of every point of $\mathcal{C}^{2}$, and the result is established.

The example $E=\left\{\left(z_{1}, 0\right): z_{1} \in \mathbb{C}\right\}$ shows that the result just derived cannot be obtained under the hypothesis that $\Lambda^{2}\left(E \cap r \mathrm{~B}_{2}\right) \leq \pi r^{2}$. It seemes probable that if $\Lambda^{2}\left(E \cap r B_{2}\right)<\pi r^{2}$ for all large values of $r$ then $E$ is removable, but no proof has presented itself. The discrepancy between $\frac{\pi^{2}}{4 \sqrt{2}}$ here arises in part from the integral geometric inequality (2) and in part from Lemma 2.

\section{A result on the ball}

We now turn to a result on the ball that is an analogue in the Bergman geometry of the result we obtained above for $c^{N}$.

The Bergman kernel on the ball in $\mathbb{C}^{N}$ is given by

$$
K(z, \zeta)=\frac{N !}{\pi^{N}} \frac{1}{(1-\langle z, \zeta\rangle)^{N+1}}
$$

if $\langle$,$\rangle denotes the Hermitian inner product on \mathcal{C}^{N}$, and the Bergman metric is given by

$$
d s^{2}=\sum_{j, k=1}^{N} T_{j k} d z_{j} \otimes d \bar{z}_{k}
$$


with coefficients $T_{j k}$ given by

$$
\begin{aligned}
T_{j k} & =\frac{\partial^{2}}{\partial z_{j} \partial \bar{z}_{k}} \ln K(z, z) \\
& =N(1-\langle z, z\rangle)^{-2}\left\{(1-(z, z)) \delta_{j k}+z_{k} \bar{z}_{j}\right\}
\end{aligned}
$$

We shall denote by $\Lambda_{B}^{\alpha}$ the $\alpha$-dimensional Hausdorff measure computed with respect to the distance function on $\mathbf{B}_{N}$ derived from the Bergman metric. We shall prove the following analogue of Theorem 1.

3. Theorem. If $E \subset \mathbf{B}_{N}$ is closed set with $\Lambda_{B}^{2 N-2}(E)<\infty$, then $E$ is removable.

4. Corollary. If $E \subset \mathbf{B}_{N}$ is a subvariety of codimension one, then $E$ has infinite area, area computed with respect to the Bergman metric.

The corollary follows from the theorem, for codimnension-one subvarieties of the ball are not removable: If $V$ is such a variety, then as we can solve the second Cousin problem on $B_{N}$, there is $f \in \mathcal{O}\left(B_{N}\right)$ with $V$ as its zero set. The reciprocal of $f$ shows $V$ not to be removable.

As we shall see below, there is a straightforward calculation that shows that if $V \subset \mathrm{B}_{N}$ is a k-dimensional variety, then $\Lambda_{B}^{2 k}(V)$ is infinite.

Proof of the Theorem: The proof follows the general lines of the proof in the case of $\mathrm{C}^{N}$, but certain integral-geometric details require attention. We start with the case that $N=2$.

Let dist $_{B}(z, w)$ denote the Bergman distance between the points $z, w \in \mathrm{B}_{2}$.

Fix a point $z_{0} \in \mathrm{B}_{2}$, and define $\rho: \mathbf{B}_{2} \rightarrow[0, \infty)$ by $\rho(z)=\operatorname{dist}_{B}\left(z, z_{0}\right)$. The triangle inequality in the Bergman distance yields that $\rho$ is a Lipschitz function:

$$
\left|\rho(z)-\rho\left(z^{\prime}\right)\right| \leq \operatorname{dist}_{B}\left(z, z^{\prime}\right) .
$$

As $\rho$ satisfies a Lipschitz condition and $\Lambda_{B}^{2}(E)<\infty$, we have that

$$
\infty>\Lambda_{B}^{2}(E)>\text { const. } \int_{[0, \infty)}^{*} \Lambda_{B}^{1}\left(E \cap\left\{z \in \mathrm{B}_{2}: \rho(z)=t\right\}\right) d t
$$

This yields a sequence $\left\{t_{1}\right\}_{i=1}^{\infty}$ with $t_{j} \rightarrow \infty$ and with

$$
\Lambda_{B}^{\prime}\left(E \cap\left\{z \in \mathbf{B}_{2}: \rho(z)=t_{j}\right\}\right) \rightarrow 0,
$$

and this implies that

$$
\Lambda^{1}\left(E \cap\left\{z \in B_{2}: \rho(z)=t_{j}\right\}\right) \rightarrow 0 .
$$


(NB. As before, $\Lambda^{1}$ denotes the 1dimensional Hausdorff measure computed with respect to the Euclidean metric.)

Let $D\left(t, z_{0}\right)=\left\{z \in \mathrm{B}_{2}: \rho\left(z, z_{0}\right)=t\right\}$. This is a ball in the Bergman metric, and its boundary is smooth.

Granted that $f \in \mathcal{O}\left(\mathrm{B}_{2} \backslash E\right)$, we know that $f \mid b D\left(t_{j}, z_{\theta}\right) \backslash E$ continues holomorphically into a neighborhood of $z_{0}$, at least when $j$ is large, so the result in the two-dimensional case is obtained as before.

To make the induction step work as before, we need two facts. First, we need to know that if $E \subset \mathbf{B}_{N+1}$ satisfies $\Lambda_{B}^{2 N}(E)<\infty$, then for almost every $\Pi \in \mathcal{G}_{N+1, N}, \Lambda_{B}^{2 N-2}(\Pi \cap \tilde{E})<\infty$ where we denote by $\tilde{E}$ the set $E \cap\{z$ : $\left.\operatorname{dist}_{B}(z, 0)>1\right\}$. $\left(\Lambda_{B}^{2 N-2}(I \cap \cap E)\right.$ denotes the Hausdorff measure computed with respect to the Bergman metric on $B_{N+1}$.) The second point we need is that the finiteness of the quantity $\Lambda_{B}^{2 N-2}(\Pi \cap E)$ implies the finiteness of the $(2 N-2)$-dimensional Hausdorff measure of the set $\Pi \cap E$ computed with respect to the Bergman metric on the $N$-dimensional ball $\Pi \cap \mathrm{B}_{N+1}$.

The latter point is straightforward though, for the metric induced on $\Pi \cap$ $\mathbf{B}_{N+1}$ from the Bergman metric on $\mathbf{B}_{N+1}$ differs only by a constant factor from the Bergman metric on the $N$-ball II $\cap \mathrm{B}_{N+1}$.

That $\Lambda_{B}^{2 N}(E)<\infty$ implies $\Lambda_{B}^{2 N-2}(\Pi \cap \tilde{E})<\infty$ for almost all $\Pi$ 's is an analogue in the Bergman metric of the result of Shifman used above. We prove the following integral-geometric fact.

5. Lemma. There is a constant $c_{N}$ such that if $S \subset \mathbf{B}_{N} \backslash\left\{z:\right.$ dist $_{B}(z, 0)<$ $1\}$, then

$$
c_{N} \Lambda_{B}^{2 N-2}(S) \geq \int_{G_{N, N-1}} \Lambda_{B}^{2 N-4}(S \cap \Pi) d \mu(\Pi) .
$$

The proof of this lemma follows precisely the lines of the proof of Shiffman's Lemma 5 in [12] once we have the following estimate.

6. Lemma. There is a constant $k_{N}$ such that for small $\delta>0$ if $T \subset \mathrm{B}_{N} \backslash\{z$ : dist $\left._{B}(0, z)<1\right\}$ and $T$ has diameter less than $\delta$ in the Bergman distance, then $\mu\left(\left\{\Pi \in \mathcal{G}_{N, N-1}: \Pi \cap T \neq \emptyset\right\}\right)<k_{N} \delta^{2}$.

For the convenience of the reader, we recall the argument in [12] that proves Lemma 5. Denote by $\delta(E)$ the diameter of the subset $E$ of $B_{N}$ computed with respect to the Bergman distance. If $E \subset \mathbf{B}_{N}$, then

$$
\int_{\mathcal{G}_{N, N-1}}^{*} \delta^{2 N-4}(E \cap \Pi) d \mu(\Pi) \leq \delta^{2 N-4}(E) \mu\{\Pi: \Pi \cap E \neq \emptyset\} .
$$

If $E \subset \mathbf{B}_{N} \backslash\left\{z: \operatorname{dist}_{B}(z, 0)<1\right\}$, and if $\delta(E)$ is small, then Lemma 6 implies the estimate

$$
\mu(\{\Pi: \Pi \cap E \neq \emptyset\}) \leq \text { const. } \delta^{2}(E),
$$


so, for such an $E$, we have

$$
\int^{*} \delta^{2 N-4}(E \cap \Pi) d \mu(\Pi)<\text { const. } \delta^{2 N-2}(E) .
$$

Now given $S$ as in Lemma 6 , assume $\Lambda_{B}^{2 N-2}(S)<\infty$. Fix a small $\varepsilon>0$, and choose a covering of $S$ by a sequence $\left\{S_{n}\right\}_{n=1}^{\infty}$ of sets with $\delta\left(S_{n}\right)<\varepsilon$ and

$$
\sum_{n} \delta^{2 N-2}\left(S_{n}\right)<\Lambda_{B, \varepsilon}^{2 N-2}(S)+\varepsilon
$$

Here

$$
\Lambda_{B, \epsilon}^{2 N-2}(S)=\inf \left\{\sum_{n} \delta^{2 N-2}\left(Q_{n}\right): S \subset \cup Q_{n} \text { and } \delta\left(Q_{n}\right)<\varepsilon\right\} .
$$

We have then that

$$
\begin{aligned}
\int_{\mathcal{G}_{N, N-1}}^{*} \Lambda_{B}^{2 N-4}(S \cap \Pi) d \mu(\Pi) & \leq \int_{\mathcal{G}_{N, N-1}}^{*} \sum_{n} \Lambda_{B}^{2 N-4}\left(S_{n} \cap \Pi\right) d \mu(\Pi) \\
& \leq \sum_{n} \int_{G_{N, N-1}^{*}} \Lambda_{B}^{2 N-4}\left(S_{n} \cap \Pi\right) d \mu(\Pi) \\
& \leq \text { const. } \sum_{n} \int_{\mathcal{G}_{N, N-1}}^{*} \delta^{2 N-4}\left(S_{n} \cap \Pi\right) d \mu(\Pi) \\
& \leq \text { const. } \sum_{n} \delta^{2 N-2}\left(S_{n}\right) \\
& \leq \text { const. }\left(\Lambda_{B_{t} \varepsilon}^{2 N-2}(S)+\varepsilon\right) .
\end{aligned}
$$

As this is true for all $\varepsilon$ and as $\Lambda_{B}^{2 N-2}(S)=\lim _{\varepsilon} \Lambda_{B, \varepsilon}^{2 N-2}(S)$, we have the desired inequality.

Lemma 6 is a consequence of the corresponding Hermitian result. The Bergman diameter of a set is not smaller than the Euclidean diameter. Thus, if $T$ has small Bergman diameter $d$ and is included in $\mathbf{B}_{N} \backslash\left\{z: \operatorname{dist}_{B}(0, z)<1\right\}$, then $T$ is contained in a Etuclidean ball $B$ of Euclidean diameter $2 d$. As $d$ is small, $B$ can be choose to lie in $\{z:|z|>1-d\}$. Everything follows from the estimate:

$$
\mu\left(\left\{\Pi: \Pi \cap \mathrm{B}\left(p_{0}, R\right\rangle \neq \emptyset\right\}\right) \leq \mathrm{const.}\left(\frac{R}{\left|p_{0}\right|}\right)^{2},
$$

which is established in the next section.

It is worth noting that our Theorem 3 implies Shiffman's result that for domains in $\mathbb{C}^{N}$, closed sets of vanishing $(2 N-2)$-dimensional measure are removable. Shifman's result is local, and if $\Lambda^{2 N-2}(E)=0$, then for every $p \in E$ and every ball $\mathbf{B}_{N}(p, r)$ centered at $p$, the set $E \cap \mathbf{B}_{N}(p, r)$ has zero $(2 N-2)$-dimensional measure with respect to the Bergman metric on $\mathbf{B}_{N}(p, r)$. Thus, $E$ is locally removabie and so removable. 


\section{An integral-geometric computation}

In the analysis above, we need to know the measure of the set of $(N-1)$ dimensional subspaces of $C^{N}$ that meet a ball. In (4) we stated an estimate that suffices; in this section, we shall evaluate this volume precisely. We shall, in fact, work in a slightly more general context. (It seems probable that the result obtained here exists somewhere in the published literature, but we know no reference.)

We are denoting by $\mathcal{G}_{N, k}$ the Grassmannian of all $k$-dimensional complex subspaces of $C^{N}$. (Thus, the elements of $\mathcal{G}_{N, k}$ pass through the origin). The manifold $\mathcal{G}_{N, k}$ is a homogeneous space of the unitary group $\mathcal{U}(N)$ : If $g \in$ $\mathcal{U}(N)$ and $\Pi \in \mathcal{G}_{N, k}$, then $g \cdot \Pi=g(\Pi) \in \mathcal{G}_{N, k}$. There is a unique measure $\mu_{k}$ on $\mathcal{G}_{N, k}$ with $\mu_{k}\left(\mathcal{G}_{N, k}\right)=1$ that is invariant under the action of $U(N)$. If we denote by $\Pi_{0}$ the element,

$$
\left\{z \in \mathbb{C}^{N}: z_{k+1}=\cdots=z_{N}=0\right\}
$$

of $\mathcal{G}_{N^{\prime}, k}$ and if $\pi: \mathcal{U}(N) \rightarrow \mathcal{G}_{N_{1} k}$ is the map given by $\pi g=g \cdot \Pi_{0}$, then $\mu_{k}$ can be calculated by

$$
\mu_{k}(E)=v\left(\pi^{-1}(E)\right)
$$

if $v$ denotes the normalized Haar measure on $U(N)$.

Our problem, precisely formulated, is the following: To determine

$$
\mu_{k}\left(\left\{\Pi \in \mathcal{G}_{N, k}: \Pi \cap \boldsymbol{B}_{N}\left(z_{0}, R\right) \neq \emptyset\right\}\right),
$$

or, equivalently, to determine

$$
v\left(\left\{g \in \mathcal{U}(N): g\left(\Pi_{0}\right) \cap \mathrm{B}_{N}\left(z_{0}, R\right) \neq \emptyset\right\}\right) .
$$

Here, $z_{0} \in \mathrm{C}^{N}$ and $R>0$. If $|R|>z_{0}$, then $0 \in \mathrm{B}_{N}\left(z_{0}, R\right)$, so the measure in question is one. In general, the answer will be a function of $z_{0}$ and $R$. The problem is plainly invariant under the action of $\mathcal{U}(N)$, so without loss of generality, we may suppose that $z_{0}=\boldsymbol{\rho}=(\rho, 0, \ldots, 0)$ with $\rho=\left|z_{0}\right|$.

We have that $g\left(\Pi_{0}\right) \cap \mathbf{B}_{N}(\boldsymbol{\rho}, R) \neq 0$ if and only if the distance $d\left(\boldsymbol{\rho}, g\left(\Pi_{0}\right)\right)$ is less than $R$.

We denote by $\left\{e_{1}, \ldots, e_{N}\right\}$ the standard orthonormal basis for $C^{N}$. Then $\left\{e_{t}, \ldots, e_{k}\right\}$ is an orthonormal basis for $\Pi_{0}$ and $\left\{e_{k+1}, \ldots, e_{N}\right\}$ is an orthonormal basis for the orthogonal complement, $\Pi_{0}^{\frac{1}{0}}$, of $\Pi_{0}$. Consequently, if $\langle$, denotes the standard Hermitian inner product on $C^{n}$ then

$$
\begin{aligned}
d\left(\rho, g\left(\Pi_{0}\right)\right) & =\left(\sum_{j=k+1}^{N}\left|\left\langle\boldsymbol{\rho}, g\left(e_{j}\right)\right\rangle\right|^{2}\right)^{\frac{1}{2}} \\
& =\left(\sum_{j=k+1}^{N} \mid\left\langle g^{-1} \rho, e_{j}\right\rangle^{2}\right)^{\frac{1}{2}} .
\end{aligned}
$$


We identify $U(N)$ with the group of unitary $N \times N$ matrices $U=\left(a_{r, s}\right)_{r, s=1, \ldots, N}$. If under this identification $g$ corresponds to $U$, then

$$
\left\langle g^{-1} \rho, e_{j}\right\rangle=\rho \bar{a}_{1 j},
$$

so

$$
d\left(\boldsymbol{\rho}, g\left(\Pi_{0}\right)\right)=\left(\sum_{j=k+1}^{N} \rho^{2}\left|a_{1 j}\right|^{2}\right)^{\frac{1}{2}}
$$

If we set

$$
\mathcal{E}(k ; c)=\left\{g \in \mathcal{U}(N): \sum_{j=1}^{N}\left|a_{1 j}\right|^{2}<c\right\}
$$

then we have to determine $v(\mathcal{E}(k ; c))$. For $c>1, \mathcal{E}(k ; c)=\mathcal{U}(N)$; in general it is an open set.

For the computation of $v(\mathcal{E}(k ; c))$ we need to recall the explicit form of the measure $v$. An invariant volume form on $U(N)$ is the form $\Omega$ given by

$$
\Omega=\left(\bigwedge_{1 \leq i<j \leq N} \omega_{i j} \wedge \bar{\omega}_{i j}\right) \wedge \bigwedge_{1 \leq k \leq N} \omega_{k k}
$$

where

$$
\omega_{i j}=\sum_{k=0}^{N} \bar{a}_{k i} d a_{k j} .
$$

The forms $\omega_{i j}$ are left-invariant on $\mathcal{U}(N)$. For the construction of $\Omega$, see [9]. In particular, one finds there the evaluation

$$
\int_{U(N)} \Omega=\prod_{j=0}^{N=1} \frac{(2 \pi i)^{j+1}}{j !} .
$$

We shall denote this value by $v(N)$. It follows then that the normalized Haar measure $v$ on $U(N)$ is the measure derived from the form $v(N)^{-1} \Omega$.

Introduce the forms $\omega^{\prime}(z)$ and $\omega(\bar{z})$ on $C^{N}$ by

$$
\omega^{\prime}(z)=\sum_{j=1}^{N}(-1)^{j-1} z_{i} d z_{1} \wedge \cdots \wedge[j] \wedge \cdots \wedge d z_{N}
$$

and

$$
\omega(\bar{z})=d \bar{z}_{1} \wedge \cdots \wedge d \bar{z}_{N}
$$

If $T: C^{N} \rightarrow C^{N}$ is a linear transformation, then $T^{*} \omega(\bar{z})=(\overline{\operatorname{det} T}) \omega(\bar{z})$ and $T^{*} \omega^{\prime}(z)=(\operatorname{det} T) \omega$. Consequently, the form $\tilde{\omega}(z)=\omega^{\prime}(z) \wedge \omega(\vec{z})$ is unitarily invariant. 
We define a map $\eta: \mathcal{U}(N) \rightarrow S^{2 N-1}$ by $\eta g=g 1$ where by 1 we mean the north pole $1=(1,0, \ldots 0)$. The fiber $\eta^{-1}(1)$ is the subgroup of $U(N)$ isomorphic to $U(N-1)$ that consists of the matrices of the form

$$
\left[\begin{array}{cccc}
1 & 0 & \ldots & 0 \\
0 & a_{22} & \ldots & a_{2 N} \\
\vdots & \vdots & \ddots & \vdots \\
0 & a_{N 2} & \ldots & a_{N N}
\end{array}\right]
$$

The form $\tilde{\omega}$ is invariant under the action of $\mathcal{U}(N)$ on $S^{2 N-1}$, and it follows that $\eta^{*} \tilde{\omega}$ is a left-invariant form on $\mathcal{U}(N)$.

At the identify of $\mathcal{U}(N)$, we have

$$
\eta^{*} \tilde{\omega}=d \alpha_{21} \wedge \cdots \wedge d \alpha_{N 1} \wedge d \bar{\alpha}_{11} \wedge d \bar{\alpha}_{21} \wedge \cdots \wedge d \bar{\alpha}_{N 1},
$$

and

$$
\begin{aligned}
\left(\bigwedge_{i<j \leq N}\left(\omega_{1 j} \wedge \bar{\omega}_{1 j}\right) \wedge \omega_{11}\right. & =\left\{\bigwedge_{1<j \leq N}\left(\sum_{k=1}^{N} \delta_{k 1} d \alpha_{k j}\right) \wedge\left(\sum_{k=1}^{N} \delta_{k 1} d \bar{\alpha}_{k j}\right)\right\} \wedge d \alpha_{11} \\
& =\left(\bigwedge_{1<j \leq N}\left(d \alpha_{1}, \wedge d \bar{\alpha}_{1 j}\right)\right) \wedge d \alpha_{11}
\end{aligned}
$$

For a unitary matrix $A$, we have $\bar{A}^{r} A=1$, i.e.,

$$
\sum_{r=1}^{N} \bar{a}_{r k} a_{r j}=\delta_{j k}
$$

whence

$$
0=\sum_{r=1}^{N} \bar{a}_{r k} d a_{r j}+\sum_{r=1}^{N} a_{r j} d \bar{a}_{r k} .
$$

Thus, $\omega_{k j}=-\bar{\omega}_{j k}$. In particular, at the identity, $d \alpha_{1 j}=-d \bar{\alpha}_{j 1}$. This implies that at the identity

$$
\left(\bigwedge_{1<j \leq N}\left(\omega_{1 j} \wedge \bar{\omega}_{1 j}\right)\right) \wedge \omega_{11}=-\left(\bigwedge_{1<j \leq N} d \bar{\alpha}_{j 1} \wedge d \alpha_{j 1}\right) \wedge d \bar{\alpha}_{11}
$$

We see then that for a suitable choice of constant $\epsilon_{N}= \pm 1$, at the identity of $\mathcal{U}(N), \eta^{*} \tilde{\omega}$ and $\epsilon_{N}\left(\bigwedge_{1<j \leq N}\left(\omega_{1 j} \wedge \bar{\omega}_{1 j}\right)\right) \wedge \omega_{11}$ coincide. As each is left invariant, they coincide on the whole $\mathcal{U}(N):$ on $\mathcal{U}(N)$,

$$
\eta^{*} \tilde{\omega}=\epsilon_{N}\left(\bigwedge_{1<j \leq N}\left(\omega_{1 j} \wedge \ddot{\omega}_{1 j}\right)\right) \wedge \omega_{11} .
$$


We now proceed to the computation of $v(\mathcal{E}(k ; c))$. For this purpose, it is convenient to notice that if $\mathcal{E}^{\prime}(k ; c)=\left\{q \in \mathcal{U}(N): \sum_{j=k+1}^{N}\left|a_{j 1}\right|^{2}<c\right\}$ then $v\left(\mathcal{E}^{\prime}(k ; c)\right)=v(\mathcal{E}(k ; c))$. Under the projection $\eta, \mathcal{E}^{\prime}(k ; c)$ goes onto the subset

$$
\sum(k ; c)=\left\{z \in S^{2 N-1}:\left|z_{k+1}\right|^{2}+\cdots+\left|z_{N}\right|^{2}<c\right\}
$$

of $S^{2 N-1}$. It follows from Fubini's theorem-see [14] for a version suitable for our purposes-that

$$
\int_{\mathcal{E}^{\prime}\{k ; c)} \Omega= \pm \int_{\Sigma(k ; c)}\left\{\int_{\eta^{-1}(z)} \bigwedge_{2 \leq i<j \leq N} \omega_{i j} \wedge \bar{\omega}_{i j} \wedge \bigwedge_{2 \leq r \leq N} \omega_{r r}\right\} \tilde{\omega} .
$$

Each of the fibers $\eta^{-1}(z)$ is a coset of the subgroup $\eta^{-1}(1)$ of $\mathcal{U}(N)$, and accordingly, for each $z$,

$$
\int_{n-1(z)} \cdots=v(N-1)
$$

Thus,

$$
\int_{\mathcal{E}^{\prime}(k ; c)} \Omega= \pm v(N-1) \int_{\Sigma(k ; c)} \tilde{\omega} .
$$

It remains for us to evaluate the integral on the right. Let us call it $I(k ; c)$. It will be convenient to introduce the notation that for $z \in C^{N}, z^{s}=\left(z_{1}, \ldots, z_{k}\right)$, $z^{\prime \prime}=\left(z_{k+1}, \ldots, z_{N}\right)$. By Stokes's theorem we have

$$
I(k ; c)=\int_{\mathbf{8}_{N} \cap\left\{\left|z^{\prime \prime}\right|^{2}<c\right\}} d \tilde{\omega}-\int_{\mathbf{B}_{N} \cap\left\{\left|z^{\prime \prime}\right|^{2}=c\right\}} \tilde{\omega}
$$

Call the first of these integrals $I^{\prime}$, the second $I^{\prime \prime}$. If $\gamma_{N}=\frac{1}{2}\left(N^{2}-N\right)$, then

$$
\begin{aligned}
I^{\prime} & =N \int_{\mathbf{B}_{N} \cap\left\{\left|z^{\prime \prime}\right|^{2}<c\right\}} d z_{1} \wedge \cdots \wedge d z_{N} \wedge d \bar{z}_{1} \wedge \cdots \wedge d \bar{z}_{N} \\
& =(-1)^{\gamma_{N}} N \int_{\mathbf{B}_{N} \cap\left\{\left|z^{\prime \prime}\right|^{2}<c\right\}} d z_{1} \wedge d \bar{z}_{1} \wedge \cdots \wedge d z_{N} \wedge d \bar{z}_{N} \\
& =(-1)^{\gamma_{N}} N \int_{\left\{\left|z^{\prime \prime}\right|^{2}<c\right\}}\left\{\int_{\left\{\left|z^{\prime \prime}\right| z^{2}<1-\left|z^{\prime \prime}\right|^{2}\right\}} d z_{1} \wedge \cdots \wedge d \bar{z}_{k}\right\} d z_{k+1} \wedge \cdots \wedge d \bar{z}_{N} \\
& =(-1)^{\gamma_{N}} N \frac{(2 \pi i)^{k}}{k !} \int_{\left\{\left|z^{\prime \prime}\right|^{2}<c\right\}}\left(1-\left|z^{\prime \prime}\right|^{2}\right)^{k} d z_{k+1} \wedge \cdots \wedge d \bar{z}_{N} \\
& =(-1)^{\gamma_{N}} N \frac{(2 i)^{N} \pi^{k}}{k !} S_{2 k-1} \int_{0}^{\sqrt{c}}\left(1-\rho^{2}\right)^{k} \rho^{2 N-2 k-1} d \rho
\end{aligned}
$$


where $S_{2 k-1}$ denotes the area of the unit sphere in $\mathbb{R}^{2 k}$ so that $S_{2 k-1}=\frac{2 \pi^{k}}{(k-1) !}$. If we expand $\left(1-\rho^{2}\right)^{k}$ with the binomial theorem and integrate term-by-term, we reach

$$
I^{t}=(-1)^{\gamma N} N \frac{(2 i)^{N} \pi^{k}}{k !} \sum_{r=0}^{k}(-1)^{r}\left(\begin{array}{l}
k \\
r
\end{array}\right) \frac{c^{N-k+r}}{2(N-k+r)} .
$$

For $I^{\prime \prime}$ we compute as follows: On the path of integration, $\Gamma$, for the integral $I^{\prime \prime}$, we have $\left|z_{k+1}\right|^{2}+\cdots+\left|z_{N}\right|^{2}=c$, so there

$$
\sum_{r=k+1}^{N} z_{r} d \bar{z}_{r}+\bar{z}_{r} d z_{r}=0
$$

Off the set where $z_{N} \neq 0$, we can solve this for $d z_{N}$ :

$$
d z_{N}=-\bar{z}_{N}^{-1}\left(\sum_{r=k+1}^{N-1} \bar{z}_{r} d z_{r}+\sum_{r=k+1}^{N} z_{r} d \bar{z}_{r}\right)
$$

This leads to the expression

$$
\begin{aligned}
\tilde{\omega}=- & \sum_{j=1}^{N-1}(-1)^{j-1} z_{i} d z_{1} \wedge \cdots \wedge[j] \wedge \cdots \wedge d z_{N-1} \wedge\left(\bar{z}_{N}^{-1} \sum_{r=k+1}^{N-1} \bar{z}_{r} d z_{r}\right) \wedge \omega(\bar{z}) \\
& +(-1)^{N-1} z_{N} d z_{1} \wedge \cdots \wedge d z_{N-1} \wedge \omega(\bar{z}) . \\
=- & \sum_{j=r+1}^{N-1}(-1)^{j-1+N-j-1} z_{j} d z_{1} \wedge \cdots \wedge d z_{j-1} \wedge\left(\bar{z}_{N}^{-1} \bar{z}_{j} d z_{j}\right) \wedge d z_{j+1} \\
& \wedge \cdots \wedge d z_{N-1} \wedge \omega(\bar{z})+(-1)^{N-1} z_{N} d z_{1} \wedge \cdots \wedge d z_{N-1} \wedge \omega(\bar{z}) \\
= & (-1)^{N-1} \bar{z}_{N}^{-1}\left(\sum_{j=r+1}^{N} z_{j} \bar{z}_{j}\right) d z_{1} \wedge \cdots \wedge d z_{N-1} \wedge \omega(\bar{z}) \\
= & c(-1)^{N-1+\gamma_{N-1}} \bar{z}_{N}^{-1} d z_{1} \wedge d \bar{z}_{1} \wedge \cdots \wedge d z_{N-1} \wedge d \bar{z}_{N-1} \wedge d \bar{z}_{N}
\end{aligned}
$$

The path of integration in $I^{\prime \prime}$ is specified by

$$
\left|z^{z}\right|^{2}=\left|z_{1}\right|^{2}+\cdots+\left|z_{k}\right|^{2}<1-c
$$

and

$$
\left|z^{\prime \prime}\right|^{2}=\left|z_{k+1}\right|^{2}+\cdots+\left|z_{N}\right|^{2}=c,
$$

so we reach

$$
\begin{aligned}
& I^{\prime \prime}=c(-1)^{N-1+\gamma_{N-1}}\left(\int_{\left\{\left|z^{\prime}\right|^{2}<1-c\right\}} d z_{1} \wedge d \bar{z}_{1} \wedge \cdots \wedge d z_{k} \wedge d \bar{z}_{k}\right) \\
& \left(\int \bar{z}_{N}^{-1} d z_{k+1} \wedge d \bar{z}_{k+1} \wedge \cdots \wedge d z_{N-1} \wedge d \bar{z}_{N-1} \wedge d \bar{z}_{N}\right) \text {. } \\
& \left\{\left|z^{\prime \prime}\right|^{2}=c\right\}
\end{aligned}
$$


The value of the first integral on the right is $\frac{(2 \pi i)^{k}}{k !}(1-c)^{k}$. The second integral is evaluated as follows

$$
\begin{aligned}
& \int \bar{z}_{N}^{-1} d z_{k+1} \wedge d \bar{z}_{k+1} \wedge \cdots \wedge d z_{N-1} \wedge d \bar{z}_{N-1} \wedge d \bar{z}_{N} \\
= & \int_{\left.\left|z^{\prime \prime}\right|^{2}=c\right\}}\left\{\int _ { | z _ { k + 1 } ^ { 2 } | + \cdots + | z _ { N - 1 } | ^ { 2 } < c | z _ { N } | ^ { 2 } = c - | z _ { k + 1 } | ^ { 2 } - \cdots - | z _ { N - 1 } | ^ { 2 } } \left\{d z_{k+1}^{-1} \wedge d \bar{z}_{k+1} \wedge \cdots \wedge d z_{N-1} \wedge d \bar{z}_{N-1}\right.\right.
\end{aligned}
$$

For every choice of $c$, the inner integral has the value $-2 \pi i$. and

$$
\begin{aligned}
\int d z_{k+1} \wedge d \bar{z}_{k+1} \wedge & \wedge \wedge \wedge d z_{N-1} \wedge d \overline{\bar{z}}_{N-1} \\
\underbrace{}_{\left|z_{k+1}\right|^{2}+\cdots+\left|z_{N-1}\right|^{2}<\varepsilon} & \frac{(2 \pi i)^{N-1-k}}{(N-1-k) !} c^{N-1-k}
\end{aligned}
$$

Thus,

$$
I^{\prime \prime}=(-1)^{N+\gamma_{N-1}} \frac{(2 \pi i)^{N}}{k !(N-1-k) !}(1-c)^{k} c^{N-k} .
$$

The quantity we are interested in is $v(\mathcal{E}(k ; c))$, which is given by

$$
\begin{aligned}
v(\mathcal{E}(k ; c)) & =v(N)^{-1} \int_{\mathcal{E}^{\prime}(k ; c)} \Omega \\
& = \pm v(N)^{-1} v(N-1)\left[I^{\prime}(k ; c)-I^{n}(k ; c)\right] .
\end{aligned}
$$

Thus,

$$
\begin{aligned}
& v(\mathcal{E}(k ; c))= \\
& \pm N !\left(\frac{1}{2 \pi i}\right)^{N}\left[(-1)^{\gamma_{N}} N \frac{(2 i)^{N} \pi^{k}}{k !} \sum_{r=0}^{k}(-1)^{r}\left(\begin{array}{l}
k \\
r
\end{array}\right) \frac{c^{N-k+r}}{2(N-k+r)}\right. \\
&\left.\quad(-1)^{N+\gamma_{N-i}} \frac{(2 \pi i)^{N}}{k !(N-1-k) !}(1-c)^{k} c^{N-k}\right] .
\end{aligned}
$$

In the preceding section, we needed the special case of this in which $k=N-1$ and $c$ is small. we see that in this case,

$$
\begin{aligned}
& v(\mathcal{E}(N-1 ; c))= \pm \frac{N !}{\pi^{N}}\left[(-1)^{\gamma_{N}} \frac{N \pi^{N-1}}{(N-1) ! 2}+0\left(c^{2}\right)-(-1)^{N+\gamma_{N-1}} \frac{\pi^{N}}{(N-1) !} c+O\left(c^{2}\right)\right] \\
& =0(c) \text {, }
\end{aligned}
$$

and this gives us what we needed. 


\section{Concluding remarks}

The results on removable singularities we have obtained above are surely not the end of the story. The two results are of the general form: $\Omega$ is a domain in an $N$-dimensional complex manifold, $d s^{2}$ is a Hermitian metric on $\Omega$ and $\Lambda_{3}^{2 N-2}$ denotes the (2N-2)-dimensional measure derived from $d s^{2}$. In two special cases, we have that a closed set $E$ in $\Omega$ is removable provided $\Lambda_{s}^{2 N-2}(E)$ is finite. One may pose the question: What conditions on the metric $d s^{2}$ suffice for us to draw this conclusion? In particular, is it sufficient for $d s^{2}$ to be a complete Kähler metric? Do the metrics of Carathéodory or Kobayashi play a rôle here?

Another problem that arises is to stablish a projective version of the result valid for meromorphic functions. Consider the Fubini-Study metric on the complex projective space $P^{N}$. With respect to this metric, the volumes of the subvarieties of $P^{N}$ form a countable set; the volume of a variety in $P^{N}$ is, to within a normalizing constant, its degree. If $E$ is a compact subset of $P^{N}$ that has $(2 N-2)$-dimensional measure (with respect to the Fubini-Study metric) less than the smallest of the volumes of codimension-one hypersurfaces in $P^{N}$, does it follow that $E$ is removable for meromorphic functions in the evident sense that it $F$ is a function meromorphic on $P^{N} \backslash E$, then $F$ extends through $E$ to be meromorphic on the whole on $P^{N}$ ?

Another question that is suggested by what we have done is the following: If $D$ is a psetudoconvex domain in $C^{N}$, must $b D$ have dimension at least $2 N-$. 2 ? The removable singularity theorem of Shiffman implies that the Hausdorff dimension or metric dimension is at least $2 N-2$, and our Theorem 1 implies that $b D$ must have infinite $(2 N-2)$-dimensional measure. The present question understands dimension in the sense of the topological theory of dimension for which one may consult [5].

\section{Appendix}

\section{The Bergman area of varieties.}

We now take up a matter to which we adverted above, the fact that subvarieties of the ball have infinite area in the Bergman metric.

Given a domain $D$ in $C^{N}$, the Bergman metric on $D$ is given by

$$
d s^{2}=\sum_{i, j=1}^{N} T_{i j} d z_{i} \otimes d \bar{z}_{j}
$$

with

$$
T_{i j}=\frac{\partial^{2}}{\partial z_{1} \partial \bar{z}_{j}} \log K(z, z)
$$


if $K$ denotes the Bergman kernel function. The associated fundamental form is the $(1,1)$-form $\omega$ given by

$$
\omega=\sum_{i, j} T_{i j} d z_{i} \wedge d \bar{z}_{j}=\partial \bar{\partial} \log K(z, z),
$$

and if $V \subset D$ is a $k$-dimensional variety, then the Bergman volume of $V$ is given by the integral $\left(\frac{1}{2 i}\right)^{k} / V \omega^{k}$.

We fix a bounded domain $V_{0} \subset V$ with $b V_{0}$ smooth enough that Stokes's theorem holds on it. Then

$$
\begin{aligned}
\int_{V_{0}} \omega^{k} & =\int_{V_{0}} d\left\{(\bar{\partial} \log K(z, z)) \wedge\left(\partial \bar{\partial} \log K(z, z)^{k-1}\right\}\right. \\
& =\int_{b V_{0}} \bar{\partial} \log K(z, z) \wedge(\partial \bar{\partial} \log K(z, z))^{k-1}
\end{aligned}
$$

We have

$$
\partial \bar{\partial} \log K=\frac{K \partial \bar{\partial} K-\partial K \wedge \bar{\partial} K}{K^{2}} .
$$

As the exterior product of a 1 -form with itself is zero, we find that

$$
\int_{b V_{0}} \bar{\partial} \log K \wedge(\partial \bar{\partial} \log K)^{k-1}=\int_{b V_{0}} K^{-k} \bar{\partial} K \wedge(\partial \bar{\partial} K)^{k-1}
$$

If $V_{r}=\{z \in D: K(z, z)<r\}$, then we find

$$
\begin{aligned}
\int_{b V_{R}} \omega^{k} & =r^{-k} \int_{b V_{r}} \bar{\partial} K \wedge(\partial \bar{\partial} K)^{k-1} \\
& =r^{-k} \int_{V_{r}}(\partial \bar{\partial} K)^{k} .
\end{aligned}
$$

In the case of the ball, where $K$ is given by (3), a computation shows that with $c_{N}=\frac{N !}{\pi^{N}}$,

$$
\bar{\partial} K \wedge(\partial \bar{\partial} K(z, z))^{k-1}=\frac{c_{N}^{k}(N+1)^{k}}{(1-\langle z, z\rangle)^{k(N+2)}}\left(\sum_{j=1}^{N} z_{j} d \bar{z}_{j}\right) \wedge\left(\sum_{j=1}^{N} d z, \wedge d \bar{z}_{j}\right)^{k-1} .
$$

If $W_{r}=\{z \in V:|z|<r\}$, then on $b W_{r}, K(z, z)=c_{N}\left(1-r^{2}\right)^{-(N+1)}$, so

$$
\begin{aligned}
\int_{\partial W_{r}} \omega^{k} & =(N+1)^{k}\left(1-r^{2}\right)^{-k} \int_{W_{r}}\left(\sum d z_{i} \wedge d \bar{z}_{i}\right)^{k} \\
& =(2 i)^{k}(N+1)^{k}\left(1-r^{2}\right)^{-k} \Lambda^{2 k}\left(W_{r}\right),
\end{aligned}
$$

where, as before, $\Lambda^{2 k}$ denotes the $2 k$-dimensional Hausdorff measure computed with respect to the Euclidean metric.

We have reached the result that if $V \subset B_{N}$ is a $k$-dimensional variety, then

$$
\Lambda_{B}^{2 k}\left(V \cap r \mathrm{~B}_{N}\right)=\frac{(N+1)^{k}}{\left(1-r^{2}\right)^{k}} \Lambda^{2 k}\left(V \cap r \mathrm{~B}_{N}\right)
$$

In particular, $V$ has infinite volume in the Bergman metric.

*A discussion of a version of Stokes's theorem sufficient for our present needs is given in [13] 


\section{References}

1. H. Alexander, Projections of polynomial hulls, J. Funct. Analysis 13 (1973), 13-16. MR45\#3757.

2. BuRAGo, YU.D., AND V.A. ZALGAller, "Geometric Inequalities," Die Grundlehren der mathematischen Wissenschaften, vol. 285, Springer-Verlag, Berlin, Heidelberg, New York, 1988. xiv + 331 pp.

3. B. CACCIOP POLI, Sul prolungamento analitico delle funzioni di due variabili complesse, Boll. Un. Mat. Ital. (I) 13 (1934), 209-212. Opere, vol. 2, 1433-145.

4. H. FEDERER, "Geometric Measure Theory," Die Grundlehren der mathematischen Wissenchaften, vol. 153, Springer-Verlag, 1969. xiv +670 pp.

5. W. Hurewicz and H. Wallman, Dimension Theory, Princeton University Press, Princeton, 1948.

6. C. LAURENT-ThIEBAUT, Sur l'extension des fonctions $\mathrm{CR}$ dans une variété de Stein, Ann. Mat. Pura Appl, (IV) 150 (1988), 141-151.

7. G. LUPACCIOLU, A theorem on holomorphic extension of CR-functions, Pacific J. Math. 124 (1986), 177-191.

8. J.-P. ROSAY AND E.L. STOUT, Radó's theorem for CR-functions, Proc. Amer. Math. Soc, to appear.

9. L.A. SAnTaló, Integral geometry in Hermitian spaces, Amer. J. Math. 74 (1952), 423-434.

10. I. R. Shaferevich, "Basic Algebraic Geometry," Die Grundlehren der mathematischen Wissenschaften, vol. 213, Springer.'Verlag, New York, Heidelberg, Berlin, 1974. xv +439 pp.

11. B. ShIFFMan, On the Removal of Singularities in Several Complex Variables, $P h . D$. dissertation, University of California, Berkeley, 1968. 51 pp.

12. B. Shiffman, On the removal of singularities of analytic functions, Mich. Math. J. 15 (1968), 111-120. MR37\#464

13. G. STOlzenberg, "Volumes, Limits and Extensions of Analytic Varieties," Lecture Notes in Mathematics, vol. 19, Springer-Verlag, Berlin, Heidelberg, New York, 1966.

14. R. SUlANKe AND P. WinTGEN, "Differentialgeometrie und Faserbündle," Birkhaüser Verlag, Basel, 1972. 
15. N. SiBony, "Les valeurs au bord de fonctions holomorphes et ensembles polynomialement convexes," Seminaire Pierre Lelong (Analysis) Année 1975/76. Lecture Notes in Mathernatics, vol 578, Springer-Verlag, Berlin, Heidelberg, New York, 1977, pp. 300-313.

The University of Washington

Department of Mathematics

Seattle, Washington 98195 USA

Rebut el 14 de Mars de 1989. 